\title{
Microfabricated Nanotopological Surfaces for Study of Adhesion-Dependent Cell Mechanosensitivity
}

\author{
Weiqiang Chen, Yubing Sun, and Jianping Fu*
}

\begin{abstract}
Cells exhibit high sensitivity and diverse responses to the intrinsic nanotopography of the extracellular matrix through their nanoscale cellular sensing machinery. A simple microfabrication method for precise control and spatial patterning of the local nanoroughness on glass surfaces by using photolithography and reactive ion etching is reported. It is demonstrated that local nanoroughness as a biophysical cue could regulate a diverse array of NIH/3T3 fibroblast behaviors, including cell morphology, adhesion, proliferation, migration, and cytoskeleton contractility. The capability to control and further predict cellular responses to nanoroughness might suggest novel methods for developing biomaterials mimicking nanotopographic structures in vivo for functional tissue engineering.
\end{abstract}

\section{Introduction}

Living cells grow and function in tissues where they are tightly associated with a three-dimensional porous network of scaffolding known as the extracellular matrix (ECM). Individual components and the architecture of the ECM and the cellular structures regulating cell-ECM interactions exhibit abundant nanoscale features that contribute to cell-ECM signaling. ${ }^{[1-3]}$ The ECM consists predominantly of interwoven proteins such as collagen, elastin, fibronectin, vitronectin, and laminin acting as adhesive ligands with dimensions ranging from a few nanometers to hundreds of nanometers. ${ }^{[4]}$ Integrins, the transmembrane receptors directly linking the ECM to the intracellular actin cytoskeleton (CSK), are the most important cell adhesion molecules and are on the scale of tens of nanometers as well. ${ }^{[4]}$ In recent years, increasing evidence has indicated that the cellular sensory machinery is

\section{W. Chen, Y. Sun, Prof. J. Fu}

Integrated Biosystems and Biomechanics Laboratory

Department of Mechanical Engineering

University of Michigan

Ann Arbor, MI 48105, USA

E-mail: jpfu@umich.edu

Prof. J. Fu

Department of Biomedical Engineering

University of Michigan

Ann Arbor, MI 48105, USA

DOI: 10.1002/smll.201201098 capable of integrating the complex nanoscale information at the cell/ECM interface into a coherent environmental signal to regulate intracellular signaling and thus cell function. ${ }^{[1,3,5,6]}$ Therefore, the local nanoscale topography in the ECM, like nanoroughness, can provide a potent regulatory signal to regulate cellular behaviors, such as cell morphology, migratory property, cell signaling, gene expression profile, and stem cell differentiation. ${ }^{[2,7-14]}$

Study of adhesion-dependent cell mechanosensitivity to nanoscale topography is critically dependent on the fabrication methods available to generate precisely controlled nanoroughness. Electron-beam and nanoimprint lithography techniques have been broadly applied to generate surfaces with nanoscale patterns. ${ }^{[11,15]}$ However, electron-beam lithography is a serial exposure technique and thus has the inherent disadvantage of low throughput, especially when writing dense patterns over a large area. Nanoimprint lithography is a simple nanolithography process with low cost and high resolution. However, it still requires the imprint mold to be generated first using electron-beam lithography, thus inheriting the inherent low-throughput limitation. Other methods to generate nanotopographic surfaces rely on either chemical treatments of polymer and metal surfaces or chemical vapor deposition of carbon nanofibers. ${ }^{[8,9,16-19]}$ However, these approaches can require multiple and complex processes, and lack the precise control and reproducibility of nanoscale topography.

The intrinsic mechanosensitive property of adherent cells to nanoscale topography is still far from completely 
understood. Thus, the ability to robustly and reproducibly generate uniformly controlled and precisely defined surfaces presented with nanotopographic cues will be necessary for exploring the mechanosensitive property of adherent cells, which will have important implications for the developing fields of functional tissue engineering and regenerative medicine.

Herein, we propose a simple yet effective microfabrication method for precise control and spatial patterning of local nanoroughness on glass surfaces by using photolithography followed by reactive ion etching (RIE). Our method is of low cost and high throughput and can generate wafer-scale surfaces with precisely controlled and patterned nanoroughness. To illustrate the general application of our RIE-generated nanorough glass surfaces, we demonstrated that local random nanoroughness could provide a potent biophysical signal to regulate a diverse array of functions of NIH/3T3 mouse embryonic fibroblasts, including cell morphology, cell adhesion, proliferation, and migration. We further explored the potential underlying mechanotransductive signals for topography sensing, and showed that adhesion-dependent cell mechanosensitivity to nanotopography might be regulated through integrin-mediated adhesion signaling and actin cytoskeletal contractility. Thus, the integrated behavioral and intracellular responses of NIH/3T3 fibroblasts clearly demonstrated the important role of nanotopography as a potent biophysical signal in regulating cellular functions. This study could provide exciting new avenues to use RIE-generated nanorough surfaces to advance our current understanding of adhesion-dependent cell mechanosensitivity.

\section{Results and Discussion}

\subsection{Fabrication Method}

Precisely controlled and locally patterned nanoroughness was generated on silica-based glass surfaces by using photolithography followed by RIE (Figure 1A). The roughness of glass surfaces was characterized with atomic force microscopy (AFM) using the root-mean-square (RMS) roughness $R_{q}$ (see Experimental Section for details of fabrication and surface characterization of nanorough glass surfaces). The initial surface roughness $R_{q}$ of unprocessed glass wafers was about $1 \mathrm{~nm}$. Nanoscale roughness ranging from 1 to $150 \mathrm{~nm}$ on the glass surface was precisely generated when the glass wafers were processed with RIE using a mixture of $\mathrm{SF}_{6}, \mathrm{C}_{4} \mathrm{~F}_{8}$, $\mathrm{He}$, and Ar gases for different periods of time. Etching of the silica-based glass wafer was consistent with a process of the ion-enhanced chemical reaction and physical sputtering. ${ }^{[20,21]}$ Interestingly, since small concentrations of impurities such as
$\mathrm{Al}, \mathrm{K}$, and $\mathrm{Na}$ exist in the silica glass, these impurities could result in accumulations of less volatile species (such as $\mathrm{AlF}_{3}$, $\mathrm{KF}, \mathrm{NaF}$, etc.) on the glass surface during the RIE process. ${ }^{[20,21]}$ These compound clusters effectively generated the so-called "micro masking" effect that could randomly shadow the glass surface and thus result in nanoscale roughening of the glass surface during RIE. ${ }^{[20]}$ Under the same RIE conditions, the nanoroughness level of the glass surface (or $R_{q}$ ) was solely determined by the RIE process duration.

Traditional photolithography was utilized for spatially patterning of nanoroughness on the glass surface. Here, glass wafers were first spin-coated with photoresist, and the photoresist layer was then patterned by photolithography to physically expose different glass regions of various sizes and shapes for subsequent RIE etching. After the RIE process, photoresist was stripped using solvents, and the glass wafers were cleaned with distilled water and a piranha solution (4:1 $\mathrm{H}_{2} \mathrm{SO}_{4} / \mathrm{H}_{2} \mathrm{O}_{2}$ ) to remove organic residues from the glass surface. ${ }^{[22]}$ Thus, by precisely controlling photolithography and RIE, we could specify the location, shape, area, and nanoroughness level of different nanorough regions on glass substrates (Figure 1B).

\subsection{Functional Responses of $\mathrm{NIH} / 3 \mathrm{~T}_{3}$ Fibroblasts}

To explore the adhesion-dependent cell mechanosensitivity to nanotopography, experiments were performed with NIH/3T3 fibroblasts using the RIE-generated nanotopological glass surfaces. Unlike most of the nanotopographic substrates used in previous studies, which require surface functionalization with ECM proteins to promote cell adhesion, the RIE-generated nanotopological glass surfaces 
did not require additional precoating or surface functionalization with ECM protein.

NIH/3T3 fibroblasts demonstrated significant adhesion selectivity between different levels of nanoroughness on glass surfaces. After $4 \mathrm{~h}$ of culture on a glass surface patterned with differently shaped nanorough islands surrounded by smooth surfaces, single NIH/3T3 fibroblasts selectively adhered to the nanorough islands where $R_{q}=70 \mathrm{~nm}$, but not on the smooth areas where $R_{q}=1 \mathrm{~nm}$ (Figure 1B). During this selective adhesion process, NIH/3T3 fibroblasts spread to conform to the different geometries of the nanorough islands (Figure 1C). In addition, when a high concentration of single NIH/3T3 fibroblasts was seeded onto a glass surface patterned with large nanorough islands, the cells prominently attached and aggregated to the patterned nanorough islands to form cell colonies that conformed to the different geometries of the nanorough islands (Figure 1D).

We further observed that NIH/3T3 fibroblasts could exhibit distinct morphological features when plated on smooth and nanorough glass surfaces. Figure $1 E$ shows scanning electron microscopy (SEM) images of single NIH/3T3 fibroblasts $24 \mathrm{~h}$ after cell seeding. A highly branched, filopodia-rich morphology of single NIH/3T3 fibroblasts was observed on smooth glass surfaces where $R_{q}=1 \mathrm{~nm}$ (Figure 1E, top), as compared to the more confined cell morphology with fewer and shorter cytoplasmic extensions on the nanorough surface where $R_{q}=150 \mathrm{~nm}$ (Figure 1E, bottom).

We quantified adhesion selectivity, defined as the ratio of the number of cells adhered to nanorough islands to the total number of cells initially seeded, of NIH/3T3 fibroblasts to patterned nanorough islands $\left(R_{q}=70 \mathrm{~nm}\right) 4 \mathrm{~h}$ after cell seeding to be about $91 \%$, which suggests that patterned nanoroughness could serve as an effective means to control the adhesion location, cell shape or spread area, and colony geometries of NIH/3T3 cells. We further quantified the cell adhesion rate, defined as the ratio of the number of cells adhered to the glass surface to the total number of cells initially seeded, 2 and $4 \mathrm{~h}$ after cell seeding against the surface roughness $\left(R_{q}=1,50,100\right.$ and $\left.150 \mathrm{~nm}\right)$. Our results showed a great enhancement of cell attachment of NIH/3T3 fibroblasts to nanorough glass surfaces with larger $R_{q}$, at least for the first $4 \mathrm{~h}$ after cell seeding (Figure $2 \mathrm{~A}$ and $\mathrm{C}$ ).

The standard 5-ethynyl-2'-deoxyuridine (EdU) cell proliferation assay (see Experimental Section for details) was performed to characterize the effect of the nanotopological cue on cell proliferation. NIH/3T3 fibroblasts were seeded at a low density $\left(3000\right.$ cells $\left.\mathrm{cm}^{-2}\right)$ on glass substrates with different values of $R_{q}(1,50,100$, and $150 \mathrm{~nm})$. Our results in Figure $2 \mathrm{~B}$ and D show that NIH/3T3 fibroblasts proliferated more rapidly on the RIE-generated nanorough surfaces, on which the cell proliferation rate was as high as $84.3 \%$ for $R_{q}=$ $150 \mathrm{~nm}$, as compared to the cells on smooth substrate $\left(R_{q}=\right.$ $1 \mathrm{~nm}$ ) with a lower cell proliferation rate of $57.8 \%$.

We further explored whether the migratory behavior of NIH/3T3 fibroblasts would be affected by the nanotopographic cue. Live-cell time-lapse images were recorded for individual migrating NIH/3T3 fibroblasts at 5-min intervals for a total period of $20 \mathrm{~h}$. Cell migration trajectories and speeds were extracted from these images using ImageJ and
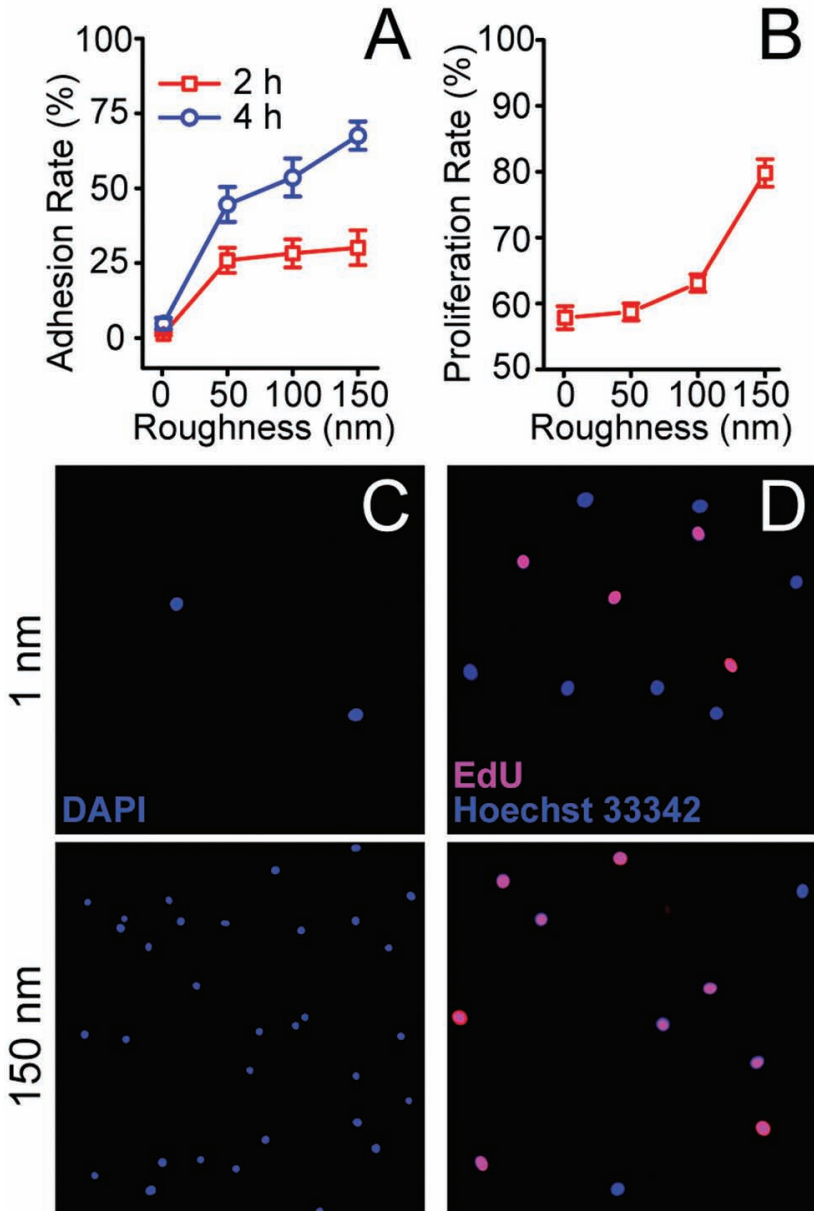

Figure 2. A) Cell adhesion rate of $\mathrm{NIH} / 3 \mathrm{~T} 3$ fibroblasts as a function of nanoroughness. Data were collected at both 2 and $4 \mathrm{~h}$ after initial cell seeding. B) Proliferation rate of NIH/3T3 cells after $8 \mathrm{~h}$ of culture as a function of nanoroughness. Data in (A) and (B) represent the means \pm standard error of the mean (s.e.m.) from three independent experiments $(n=3)$. C) Representative immunofluorescence images of DAPI-stained nuclei of NIH/3T3 fibroblasts plated on smooth $\left(R_{q}=1 \mathrm{~nm}\right.$; top) and nanorough ( $R_{q}=150 \mathrm{~nm}$; bottom) substrates after $4 \mathrm{~h}$ of culture. These images were used for calculation of the cell adhesion rate shown in (A). D) Representative immunofluorescence images of NIH/3T3 fibroblasts stained with Hoechst 33342 (blue) and EdU (pink) for enumeration of proliferating cells on smooth $\left(R_{q}=1 \mathrm{~nm}\right)$ and nanorough $\left(R_{q}=150 \mathrm{~nm}\right)$ substrates after $8 \mathrm{~h}$ of culture. These images were used for calculation of the cell proliferation rate shown in (B).

the manual object tracking plug-in MTrackJ (Figure 3A). $\mathrm{NIH} / 3 \mathrm{~T} 3$ fibroblasts showed an increased migration speed against the surface roughness (Figure 3B). For example, the migration speed of NIH/3T3 fibroblasts on the nanorough surface where $R_{q}=150 \mathrm{~nm}$ was $0.75 \mu \mathrm{m} \mathrm{min}{ }^{-1}$, about 2.7 times greater than the speed on the smooth substrate where $R_{q}=1 \mathrm{~nm}$.

Since cell adhesion and migration properties of NIH/3T3 fibroblasts were critically regulated by nanotopographic cue, we next examined whether NIH/3T3 fibroblasts would show any directional preference when crossing a topology boundary between smooth and nanorough regions patterned on the glass surface. We first observed that when NIH/3T3 fibroblasts were initially located within a nanorough island, their 


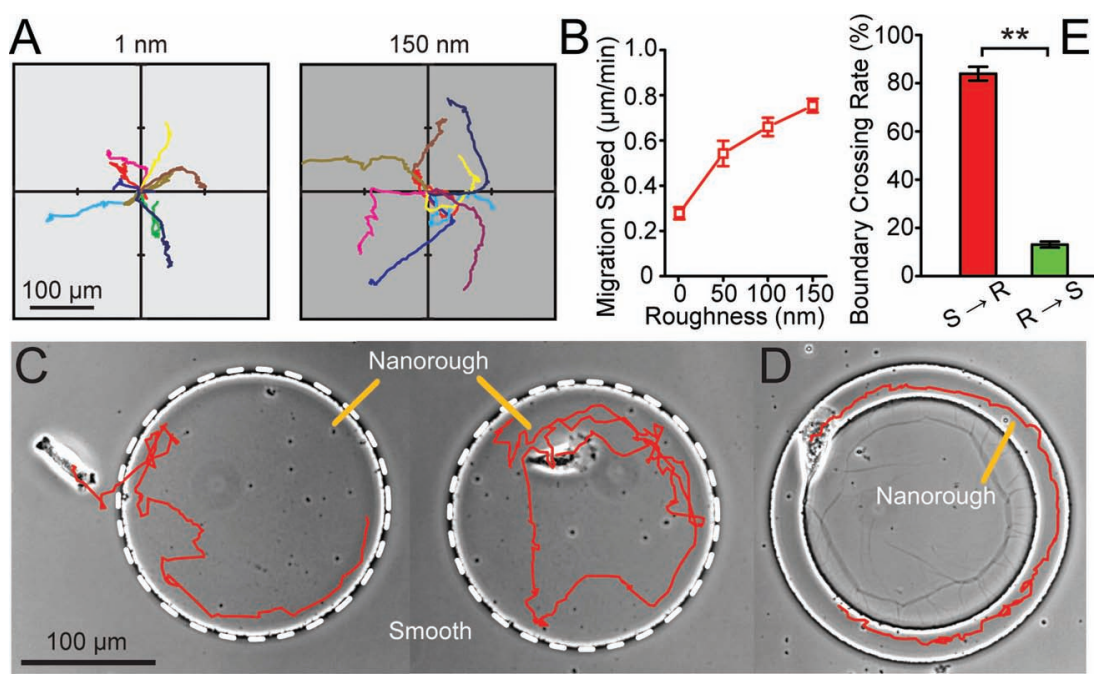

Figure 3. A) Migration trajectories of $\mathrm{NIH} / 3 \mathrm{~T} 3$ fibroblasts on smooth $\left(R_{q}=1 \mathrm{~nm}\right)$ and nanorough $\left(R_{q}=150 \mathrm{~nm}\right)$ substrates during a period of $6.5 \mathrm{~h}$ after $24 \mathrm{~h}$ of culture. These trajectories were used for calculation of cell migration speed shown in (B). B) Migration speed of $\mathrm{NIH} / 3 \mathrm{~T} 3$ cells as a function of nanoroughness. Data represent the means \pm s.e.m. from ten independent experiments $(n=10)$. C) Migration trajectories of NIH/3T3 fibroblasts that were initially either outside (left) or inside (right) a circular nanorough pattern $\left(R_{q}=70 \mathrm{~nm}\right)$. D) Migration trajectory showing a confined cell migration in a circular nanorough loop $\left(R_{q}=\right.$ $70 \mathrm{~nm}$ ). E) Boundary-crossing rate of $\mathrm{NIH} / 3 \mathrm{~T} 3$ fibroblasts, for cells approaching the topography boundary either from the smooth $\left(R_{q}=1 \mathrm{~nm}\right)$ to the nanorough $\left(R_{q}=70 \mathrm{~nm}\right)$ side $(\mathrm{S} \rightarrow \mathrm{R})$ or from the nanorough to smooth side $(R \rightarrow S)$, as indicated in the plot. Data represent the means \pm s.e.m. from five independent experiments. Statistical analysis was performed by employing Student's $t$-test. $* *$ indicates $p<0.01$.

migration trajectory could be confined within the nanorough island for a long period of time (Figure 3C and Supporting Information Figure S1). Further, when fibroblasts approached the topology boundary of a nanorough island from the nanorough side, they would most likely turn around or retract from the topology boundary and would not migrate across the boundary of the nanorough island (Figure 3C; Supporting Video V1). In contrast, fibroblasts approaching the topology boundary of a nanorough island from the smooth side could easily migrate across the boundary to enter the nanorough island (Figure 3C; Supporting Video V2). We further quantified the boundary-crossing rate, defined as the percentage of cells that successfully migrated across the topology boundary, for cells approaching the boundary either from the smooth to nanorough side ( $\mathrm{S} \rightarrow \mathrm{R}$; cross-in) or from the nanorough to smooth side ( $\mathrm{R} \rightarrow \mathrm{S}$; cross-out). The cross-in rate of NIH/3T3 fibroblasts for a nanorough pattern with $R_{q}=70 \mathrm{~nm}$ was $84 \%$, much greater than the cross-out rate, which was only 13\% (Figure 3E).

The migration preference of NIH/3T3 fibroblasts on nanorough surfaces further allowed the noninvasive control of cell migration by using nanorough patterns on the glass surface. For example, we demonstrated that the migration of $\mathrm{NIH} / 3 \mathrm{~T} 3$ fibroblasts followed a circular loop-shaped nanorough pattern (Figure 3D and Supporting Videos V3-V6). Together, our results demonstrated that the RIE-generated nanotopological cue could provide a potent regulatory signal to mediate a diverse array of NIH/3T3 fibroblast behaviors including cell morphology, adhesion, proliferation, and migration.

\subsection{Nanotopographic Regulation of Focal Adhesion Formation}

Although much effort has been focused on functional studies of the nanotopographic sensing by adherent cells, the molecular mechanism for adhesion-dependent cell mechanosensitivity to nanotopography remains largely undetermined. Existing evidence has suggested that integrin-mediated focal adhesion (FA) signaling, which is critical for many cellular functions and strongly dependent on their nanoscale molecular arrangement and dynamic organization, might play an important role in regulating cell mechanosensitivity to nanotopography. ${ }^{[3]}$

To investigate the likely involvement of integrin-mediated FA formation in regulating topological sensing of NIH/3T3 fibroblasts, we examined FA formation of single NIH/3T3 fibroblasts plated on nanorough glass surfaces. After $24 \mathrm{~h}$ of culture, single NIH/3T3 fibroblasts exhibited distinct FA formation and organization on the smooth and nanorough glass surfaces, as characterized by immunofluorescence staining of vinculin, a FA protein (Figure 4A). On the smooth glass surface where $R_{q}=1 \mathrm{~nm}$, mature and prominent vinculin-containing FAs formed primarily on the periphery of the cells. In contrast, on the nanorough surface where $R_{q}$ $=150 \mathrm{~nm}, \mathrm{NIH} / 3 \mathrm{~T} 3$ fibroblasts exhibited randomly distributed, punctate FAs of small areas throughout the entire cell spread area. Morphometric analysis of cell populations suggested that on the nanorough glass surface with $R_{q}=$ $150 \mathrm{~nm}, \mathrm{NIH} / 3 \mathrm{~T} 3$ fibroblasts had smaller mean cell spread area and total FA area per cell than the cells on the smooth surface (Figure 4D,E). Furthermore, our quantitative results indicated that NIH/3T3 fibroblasts on the nanorough surface formed FAs of smaller sizes but with a greater density as compared with the cells on the smooth surface (Figure 4E-H). These small, punctate FAs suggested rapid FA turnover and weak actomyosin CSK contractility, which could lead to disorganized actin filaments and rapid cell migration.

We further performed single-cell correlative studies by plotting single-cell data of the total FA area per cell and number of FAs per cell against cell spread area (Figure 4B,C). Figure 4B shows that all the single-cell data of the total FA area per cell collapsed and followed a single trend against cell spread area, regardless of surface roughness, thus indicating comparable total FA area per cell on both smooth and rough surfaces. However, all the single-cell data of the number of FAs per cell on smooth $\left(R_{q}=1 \mathrm{~nm}\right)$ and nanorough $\left(R_{q}=50\right.$, $100,150 \mathrm{~nm}$ ) surfaces followed different trends against cell spread area, evidenced by the different slopes of the fitting curves, thereby indicating increased FA number per cell area on nanorough surfaces (Figure 4C). 

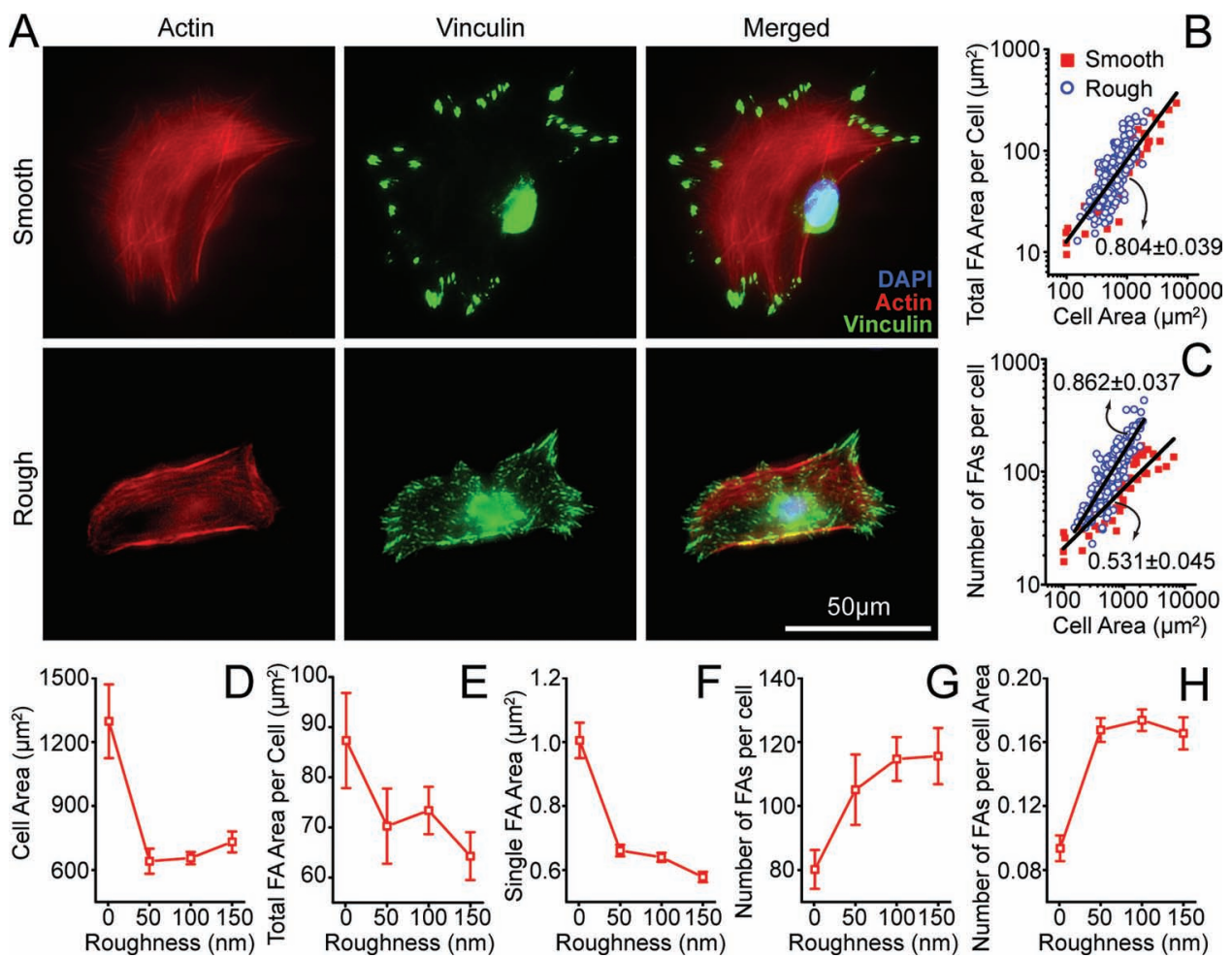

Figure 4. A) Representative immunofluorescence images of NIH/3T3 fibroblasts on smooth $\left(R_{q}=1 \mathrm{~nm}\right)$ and nanorough $\left(R_{q}=150 \mathrm{~nm}\right)$ substrates after $24 \mathrm{~h}$ of culture. Cells were co-stained for nuclei with DAPI (blue), actin (red), and vinculin (green). B-H) Quantitative and correlative analysis of cell morphology and FA formation of NIH/3T3 fibroblasts; (B) and (C) show the total FA area per cell (B) and the number of FAs per cell (C) as a function of cell area. Each data point in (B) and (C) represents an individual cell plated on either smooth $\left(R_{q}=1 \mathrm{~nm}\right.$; cell number $\left.n=50\right)$ or nanorough $\left(R_{q}=50,100,150 \mathrm{~nm}\right.$; cell number $\left.n=191\right)$ surfaces. Data trends in (B) and (C) were plotted using linear least-squares fitting (black lines), with the slope values \pm s.e.m. indicated. (D) $(\mathrm{H})$ plot cell area (D), total FA area per cell (E), average single FA area (F), total number of FAs per cell $(G)$, and number of FAs per cell area $(\mathrm{H})$ as a function of nanoroughness. Data represent the means \pm s.e.m. For each data point, cell number $n>50$.

Taken together, our comparative and correlative studies strongly indicated that FA formation and organization were tightly coupled cellular mechanosensory systems involved in transducing nanotopography signals in the local cellular microenvironment into intracellular responses. The molecular arrangement and dynamic organization of integrin-mediated FAs appeared to be sensitive and responsive to local presentation of a nanotopographical cue.

\subsection{Nanotopographic Regulation of Cytoskeletal Contractility}

The small, punctate FAs and confined cell spreading of NIH/3T3 fibroblasts on the nanorough glass surface implicated the involvement of actin CSK remodeling in adhesiondependent cell mechanosensitivity to nanotopography. ${ }^{[23]}$

To investigate the potential involvement of actin CSK remodeling in adhesion-dependent cell mechanosensitivity to nanotopography, we utilized an array of elastomeric polydimethylsiloxane (PDMS) microposts as live-cell force sensors to report subcellular traction forces exerted by cells adhered on the tops of the PDMS microposts (Figure 5). The PDMS micropost array is a well-established technique useful for cell mechanics and mechanobiology study. ${ }^{[24-26]}$ Recent studies have demonstrated that a quick RIE treatment can generate nanoscale roughness on the PDMS surface. ${ }^{[27]}$ Thus, in this work, nanoroughness was generated on the tops of the PDMS microposts using $\operatorname{RIE}\left(R_{q}=53 \mathrm{~nm}\right.$, Figure 5A; see Experimental Section for details). Our quantitative analysis of cell morphology and CSK contractility of NIH/3T3 fibroblasts revealed that the cell spread area and traction force decreased significantly for cells plated on roughened PDMS microposts as compared to the cells plated on unroughened controls (Figure 5C-F). We further performed correlative studies of single-cell data of traction force and cell spread area. Our data in Figure 5G showed strong linear correlations between traction force and cell spread area, for NIH/3T3 fibroblasts on both smooth $\left(R_{q}=0.5 \mathrm{~nm}\right)$ and nanorough $\left(R_{q}=53 \mathrm{~nm}\right)$ PDMS microposts. However, the slope of the linear correlation between traction force and cell spread area was substantially less for cells plated on the nanorough microposts than for cells on the smooth ones. Combining together the results in Figures 4 and 5 , it appeared that nanotopography might regulate the behavior of NIH/3T3 fibroblasts through its direct effect on the local molecular arrangement, and the formation and distribution of FAs that might in turn regulate the CSK organization and contractility and thus downstream cellular functions. 

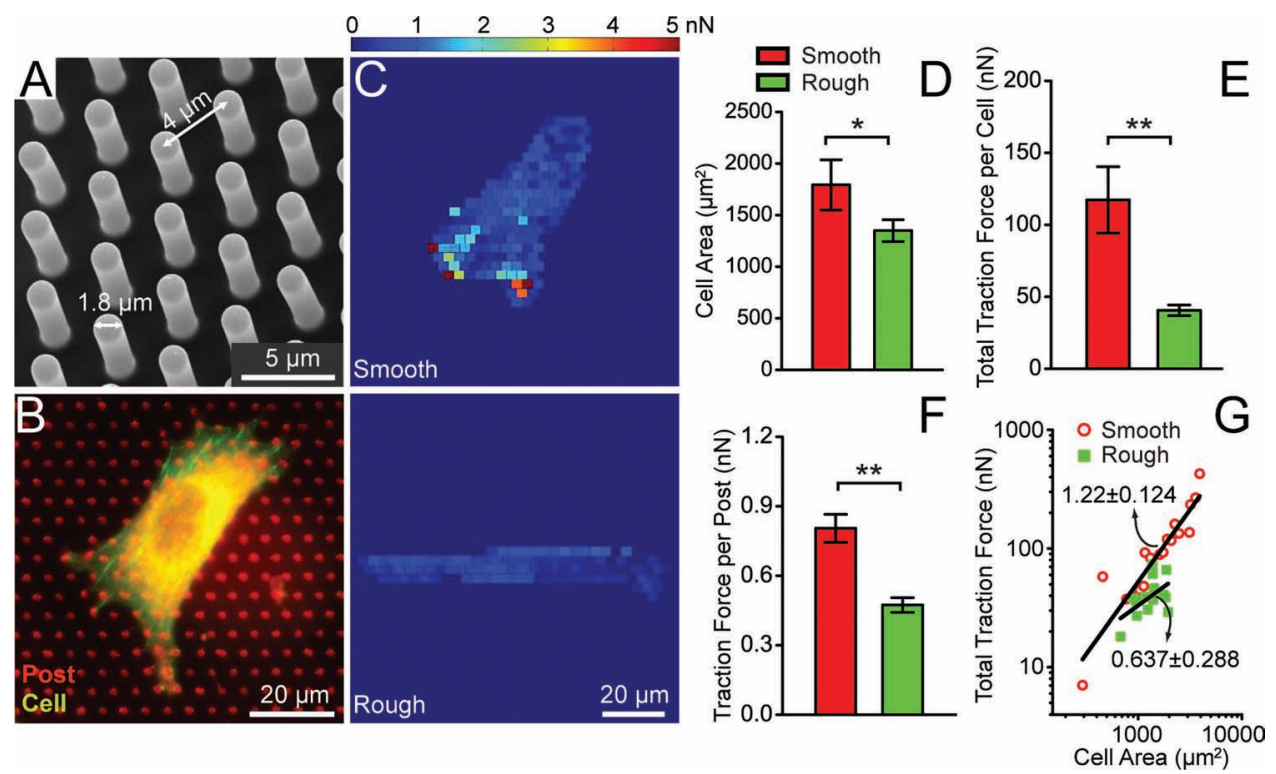

Figure 5. A) SEM image showing the PDMS micropost array with a post diameter of $1.83 \mu \mathrm{m}$, height of $8.3 \mu \mathrm{m}$, and center-to-center distance of 4 $\mu \mathrm{m}$. B) Representative immunofluorescence image showing a single NIH/3T3 fibroblast on the PDMS micropost array $24 \mathrm{~h}$ after initial cell seeding. The cell was stained with fluorophore-labeled phalloidin for actin filaments (yellow), and the underlying PDMS posts were visualized using Dil (see Experimental Section). C) Colorimetric maps showing subcellular traction forces exerted by single NIH/3T3 fibroblasts on the PDMS microposts. The PDMS microposts were either untreated (top, smooth; $R_{q}=0.5 \mathrm{~nm}$ ) or roughened using RIE (bottom, rough; $R_{q}=53 \mathrm{~nm}$ ). D-G) Quantitative analysis of cell morphology and traction force for NIH/3T3 fibroblasts. (D)-(F) plot the cell area (D), total traction force per cell (E), and traction force per post (F) for single NIH/3T3 fibroblasts plated on the PDMS microposts with either smooth $\left(R_{q}=0.5 \mathrm{~nm}\right)$ or nanorough $\left(R_{q}=53 \mathrm{~nm}\right)$ top surfaces. Data represent the means \pm s.e.m. $(n>16)$. Statistical analysis was performed by employing the Student's $t$-test. $* p<0.05$; $* * p<0.01$. $\mathrm{G})$ Total traction force per cell as a function of cell area. Each data point represents an individual cell on the PDMS microposts with either smooth $\left(R_{q}=0.5 \mathrm{~nm} ; n=19\right)$ or nanorough $\left(R_{q}=53 \mathrm{~nm} ; n=16\right)$ top surfaces. Data trends in (G) were plotted using linear least-squares fitting (black lines), with the slope values \pm s.e.m. indicated.

\subsection{Post-RIE Residues on Glass Surfaces and Their Negligible Effect on Cellular Sensitivity to Nanotopography}

It is known that RIE can leave some chemical residues on nanorough glass surfaces after the RIE etching process. ${ }^{[22]} \mathrm{To}$ confirm that the post-RIE residues on the nanorough glass surfaces would not affect the intrinsic cellular sensitivity and responses to nanotopography, we applied X-ray photoelectron spectroscopy (XPS; Kratos Axis Ultra DLD, Kratos Analytical Ltd, Manchester, UK) to examine potential chemical residues left on nanorough glass surfaces after RIE. From the XPS spectra shown in the Supporting Information (Figure S2A), we did indeed observe a peak of elemental F for RIE-processed glass surfaces, which did not show in the XPS spectrum for unprocessed flat glass surfaces. These $\mathrm{F}$ residues might have resulted from the $\mathrm{SF}_{6}$ and $\mathrm{C}_{4} \mathrm{~F}_{8}$ gases used in the RIE process.

We then examined whether the $\mathrm{F}$ residues deposited on the RIE-etched nanorough glass surfaces could have measurable effects on the normal behavior of NIH/3T3 fibroblasts, by comparing the behavior of the cells on nanorough glass substrates treated with or without brief etching with buffered hydrofluoric acid (BHF). Our results (Supporting Information Figure S2A and B) suggested that a brief treatment with BHF for $15 \mathrm{~s}$ for RIE-processed nanorough glass surfaces could effectively remove $\mathrm{F}$ residues on the glass surfaces without affecting significantly their nanoroughness. We thus compared the cell adhesion, proliferation, and FA formation of NIH/3T3 fibroblasts on glass substrates with similar nanoroughness levels that were treated with or without BHF cleaning. Our data (Supporting Information Figure S2C-G) clearly suggested that $\mathrm{F}$ residues on RIE-processed glass surfaces had negligible effects on the normal cellular behavior of NIH/3T3 cells. Thus, we concluded that cellular responses to RIE-processed nanorough glass surfaces were due to the intrinsic cellular sensitivity to the nanotopography.

\section{Conclusion}

We have reported a simple, yet effective, microfabrication strategy for the precise control and patterning of local nanoroughness on glass surfaces by using photolithography and RIE. We demonstrated that NIH/3T3 fibroblasts were intrinsically sensitive to the RIE-generated nanoscale topological cue, as evidenced by reduced cell spread area, enhanced cell adhesion, rapid cell proliferation, and cell migration on nanorough glass surfaces as compared to smooth controls. Our results further suggested that cellular responses of NIH/3T3 fibroblasts to nanotopography might be functionally linked to their disrupted FA formation and spatial reorganization of CSK structure and contractility. We provided direct experimental evidence showing that the CSK contractility of NIH/3T3 fibroblasts decreased significantly in response 
to the nanotopological cue, which might shed light on the molecular mechanism for the adhesion-dependent cell mechanosensitivity to nanotopography. We suspect that a feedback regulation and mechanochemical integration mechanism involving integrin-mediated FA, actin CSK, and intracellular mechanosensory components might play an important role in regulating the mechanosensitive behaviors of NIH/3T3 fibroblasts. Our RIE-based technique to robustly and reproducibly generate uniformly controlled and precisely defined surfaces presented with a nanotopographic cue might find potential applications in the general areas of cell-based assays and tissue engineering.

\section{Experimental Section}

Fabrication of Nanorough Glass Samples: To achieve a precise control and spatial patterning of nanoroughness on glass substrates, we developed a simple yet precise fabrication method based on photolithography and RIE. In brief, photoresist was first spincoated on glass wafers (Borofloat 33, Plan Optik, Elsoff, Germany) and patterned using photolithography to physically expose different regions of the underlying glass wafer. The patterned glass wafer was then processed with RIE (LAM 9400, Lam Research, Fremont, CA) for different periods of time to generate different levels of the nanoscale surface roughness $\left(R_{q}=1-150 \mathrm{~nm}\right)$ on the exposed regions of the glass wafer, where the photoresist had previously been developed and dissolved. The RIE processing conditions used in this work were: $\mathrm{SF}_{6}$ (8 sccm), $\mathrm{C}_{4} \mathrm{~F}_{8}$ (50 sccm), He (50 sccm), $\operatorname{Ar}(50 \mathrm{sccm})$, chamber pressure $1.33 \mathrm{~Pa}$, bias voltage $100 \mathrm{~V}$, and radio-frequency power $500 \mathrm{~W}$. The resulting RIE glass etch rate was about $50 \mathrm{~nm} \mathrm{~min}{ }^{-1}$. After the RIE process, photoresist was stripped using solvents, and the glass wafer was rinsed with distilled water. For unpatterned nanorough glass samples, bare glass wafers were directly processed with RIE using the same RIE conditions as described above. The glass wafers were cut into small pieces $(2 \mathrm{~cm} \times 2 \mathrm{~cm})$ using a die saw (ADT7100; Advanced Dicing Technologies Ltd., Yokneam, Israel) before assays with cells.

Surface Characterization Using Atomic Force Microscopy: The nanoroughness of the glass or PDMS surfaces was measured at room temperature with AFM (Veeco NanoMan atomic force microscope; Digital Instruments Inc., Santa Barbara, (A) using a noncontact tapping mode and standard Si tapping mode AFM tips. The AFM scan size was set as $10 \mu \mathrm{m} \times 10 \mu \mathrm{m}$ with a scan rate of $1 \mathrm{~Hz}$. The resulting map of the local surface height was represented by using AFM topographs. The nanoroughness of each sample was characterized using the root-mean-square (RMS) roughness $R_{q}$ of the local surface height over the whole sample area scanned by AFM. The initial surface roughness $R_{q}$ of unprocessed bare glass wafers and PDMS surfaces characterized by AFM was about 1 and $0.5 \mathrm{~nm}$, respectively.

Cell Culture and Reagents: NIH/3T3 mouse embryonic fibroblasts (ATCC, Manassas, VA) were maintained in a growth medium consisting of high-glucose Dulbecco's modified Eagle's medium (DMEM; Invitrogen, Carlsbad, CA) supplemented with $10 \%$ bovine serum (Atlanta Biological, Atlanta, GA), $100 \mu \mathrm{g} \mathrm{mL} \mathrm{L}^{-1}$-glutamine, 100 units $\mathrm{mL}^{-1}$ penicillin, and $100 \mu \mathrm{g} \mathrm{mL} \mathrm{L}^{-1}$ streptomycin. Fresh $0.25 \%$ trypsin-EDTA in phosphate-buffered saline (PBS) was used to resuspend $\mathrm{NIH} / 3 \mathrm{~T} 3$ cells. The cells were seeded at a low density
( 3000 cells $\mathrm{cm}^{-2}$ ) in the growth medium onto the glass or PDMS surfaces.

SEM Specimen Preparation: Cells were washed three times with $50 \mathrm{~mm}$ Na-cacodylate buffer (pH 7.3; Sigma-Aldrich, St. Louis, MO), fixed for $1 \mathrm{~h}$ with $2 \%$ glutaraldehyde (Electron Microscopy Sciences, Hatfield, PA) in $50 \mathrm{~mm}$ Na-cacodylate buffer, and dehydrated in a graded series of ethanol concentrations through $100 \%$ over a period of $1.5 \mathrm{~h}$. Dehydration in $100 \%$ ethanol was performed three times. Dehydrated samples were then dried with liquid $\mathrm{CO}_{2}$ using a supercritical point dryer (Samdri-PVT-3D, Tousimis, Rockville, MD). Samples were mounted on stubs, sputtered with gold/palladium, and observed and photographed by SEM (Hitachi SU8000 ultrahigh-resolution microscope; Hitachi High Technologies America, Inc., Pleasanton, CA).

EdU Cell Proliferation Assay: For the EdU cell proliferation assay, NIH/3T3 cells were first starved at confluence in the growth medium supplemented with $0.5 \%$ bovine serum (Invitrogen) for $48 \mathrm{~h}$ to synchronize the cell cycle before trypsinization. Synchronized cells were replated on the glass substrates, recovered in the complete growth medium for 12 to $24 \mathrm{~h}$, and were then exposed to $4 \mu \mathrm{M}$ 5-ethynyl-2'-deoxyuridine (EdU; Invitrogen) in the growth medium for $8 \mathrm{~h}$. Cells were then fixed with 3.7\% formaldehyde (Electron Microscopy Science) in PBS, permeabilized with $0.3 \%$ Triton X-100 (Roche Applied Science, Indianapolis, IN) in PBS, blocked with $10 \%$ goat serum, and stained with Alexa Fluor 488 conjugated azide targeting the alkyne groups in EdU, which was incorporated in newly synthesized DNA. Cells were co-stained with Hoechst 33342 (Invitrogen) to visualize the cell nucleus.

Immunofluorescence Staining: For total cell counts, cell nuclei were stained with 4',6-diamidino-2-phenylindole (DAPI; Invitrogen). For visualization of F-actin, cells were fixed with $4 \%$ paraformaldehyde (Electron Microscopy Science) in PBS. F-actin was detected with fluorophore-conjugated phalloidin (Invitrogen). Immunofluorescence staining of FAs was performed as previously described. ${ }^{[24]}$ In brief, cells were incubated in an ice-cold cytoskeleton buffer (50 m $\mathrm{m} \mathrm{NaCl}, 150 \mathrm{~mm}$ sucrose, $3 \mathrm{~mm} \mathrm{MgCl}, 1 \mu \mathrm{g} \mathrm{mL} \mathrm{L}^{-1}$ aprotinin, $1 \mu \mathrm{g} \mathrm{mL}^{-1}$ leupeptin, and $1 \mu \mathrm{g} \mathrm{mL}^{-1}$ pepstatin) for $1 \mathrm{~min}$, and then permeabilized for $1 \mathrm{~min}$ in the cytoskeleton buffer supplemented with $0.5 \%$ Triton X-100. Detergent-extracted cells were fixed with $4 \%$ paraformaldehyde in PBS for 30 min, washed with PBS, incubated with $10 \%$ goat serum (Invitrogen) for $1 \mathrm{~h}$, incubated with a primary antibody to vinculin produced in mouse (Sigma-Aldrich) for $1 \mathrm{~h}$, and stained with Alexa Fluor 488 conjugated goat antimouse immunoglobulin $\mathrm{G}$ ( $\mathrm{gg}$ ) secondary antibody (Invitrogen) for 1 h. Alexa Fluor 555 conjugated phalloidin (Invitrogen) and DAPI were used to visualize F-actin and the nucleus, respectively.

Quantitative Analysis of Cell Adhesion, Cell Spread Area, and FA: For quantitative analysis of cell adhesion, NIH/3T3 cells were seeded at a low density ( 3000 cells $\mathrm{cm}^{-2}$ ) in the growth medium onto the glass samples with different roughnesses. The cells were then incubated for 2 or $4 \mathrm{~h}$. Cells were then fixed with $4 \%$ paraformaldehyde in PBS for 30 min, washed with PBS, and incubated with DAPI for $1 \mathrm{~h}$ to visualize nuclei. Immunofluorescence images (100 images per sample) of the nuclei were taken using an epifluorescence microscope (Carl Zeiss Axio Observer Z1, Carl Zeiss Microlmaging, Thornwood, NY) equipped with a thermoelectrically cooled monochrome CCD camera (AxioCam camera; Carl Zeiss Microlmaging) and a 10× objective (0.3 NA; EC Plan NEOFLUAR; Carl Zeiss Microlmaging). ImageJ (National Institutes of Health, 
Bethesda, MD) was then used to determine the number of cells adhered to the sample surface.

Cell spread area and FA formation were quantified as previously described. ${ }^{[24,28]}$ In brief, immunofluorescence images of actin CSK and vinculin were obtained using an epifluorescence microscope (Carl Zeiss Axio Observer Z1 Carl Zeiss Microlmaging, Thornwood, NY) equipped with a thermoelectrically cooled monochrome CCD camera (AxioCam camera; Carl Zeiss Microlmaging) and a $40 \times$ objective (0.75 NA; EC Plan NEOFLUAR; Carl Zeiss Microlmaging). Images were captured using the Axiovision Software (Carl Zeiss Microlmaging) and processed using custom-developed MATLAB programs (Mathworks, Natick, MA). To determine the cell spread area, the Canny edge detection method was used to binarize the actin fibers and FAs in the images, and then image dilation, erosion, and fill operations were used to fill in the gaps between the white pixels in the images. The resultant white pixels were summed to quantify cell spread area. To quantify FA number and area, the grayscale vinculin image was thresholded to produce a black and white FA image from which the white pixels, representing FAs, were counted and summed.

Quantitative Analysis of Cell Migration: Time-lapse microscopy experiments were performed for cell migration assays. Individual cells were chosen at random, and their phase-contrast images were recorded at 5-min intervals for a total period of $20 \mathrm{~h}$ with the Carl Zeiss Axio Observer Z1 microscope using a 10x objective (0.3 NA; EC Plan NEOFLUAR; Carl Zeiss Microlmaging). The microscope was enclosed in an environmental chamber (XL S1 chamber; Carl Zeiss Microlmaging) to maintain the experimental environment at $37{ }^{\circ} \mathrm{C}$ and $5 \% \mathrm{CO}_{2}$. Cell migration trajectories and speeds were determined from the recorded microscope images using ImageJ (National Institutes of Health, Bethesda, MD) and the manual object tracking plug-in MTrackJ (developed by Dr. E. Meijering, Biomedical Imaging Group of Rotterdam, University Medical Center of Rotterdam, The Netherlands).

Fabrication of PDMS Micropost Arrays: The PDMS micropost arrays were fabricated using deep reactive ion etching (DRIE) and replica molding, as previously described. ${ }^{[24,25]}$ Briefly, silicon micropost array masters were fabricated using projection photolithography and DRIE. By controlling the mask design of the micropost array and the DRIE process time, we determined precisely the different geometrical factors of the silicon micropost array master, including post diameter, post center-to-center distance, and post height. The PDMS micropost array was then generated through a double-casting process, which ensured a planar surface of the PDMS micropost tops. ${ }^{[25]}$ The silicon masters were first silanized with (tridecafluoro-1,1,2,2,-tetrahydrooctyl)-1trichlorosilane vapor (United Chemical Technologies, Bristol, $\mathrm{PA})$ for $4 \mathrm{~h}$ under vacuum to facilitate subsequent release of the negative PDMS mold from the silicon master. PDMS prepolymer (Sylgard 184, Dow-Corning, Midland, MI) was then prepared by thoroughly mixing the monomer with the curing agent (with a $w / w$ ratio of $10: 1$ ), poured onto the silicon master, and cured at $110{ }^{\circ} \mathrm{C}$ for $20 \mathrm{~min}$. The fully cured negative PDMS mold was peeled off the silicon mold, and the excess PDMS was trimmed using a razor blade. The negative PDMS mold was then activated with an oxygen plasma for 1 min (200 mTorr; Plasma Prep II, West Chester, PA) and silanized with (tridecafluoro-1,1,2,2,tetrahydrooctyl)-1-trichlorosilane vapor for $24 \mathrm{~h}$ to facilitate subsequent release of the PDMS micropost array from the negative
PDMS mold. To generate the final PDMS micropost array, 1:10 ratio PDMS prepolymer was poured over the negative PDMS mold and degassed under vacuum for $10 \mathrm{~min}$. A $25 \mathrm{~cm} \times 25 \mathrm{~cm}$ cover glass, which served as the substrate for the PDMS micropost array, was then placed on top of the negative PDMS mold. After curing at $110{ }^{\circ} \mathrm{C}$ for $40 \mathrm{~h}$, the PDMS micropost array was peeled off the negative mold to release the final PDMS micropost array. When peeling induced collapse of the PDMS microposts, we regenerated freestanding PDMS microposts by sonication in $100 \%$ ethanol for $30 \mathrm{~s}$ followed by dry-release with liquid $\mathrm{CO}_{2}$ using a critical point dryer. The PDMS micropost array used in this study had a post diameter of $1.83 \mu \mathrm{m}$, a height of $8.3 \mu \mathrm{m}$ and a center-to-center distance of $4 \mu \mathrm{m}$.

To generate nanoscale roughness on the top surface of the PDMS microposts, the PDMS micropost array was processed with RIE for $5 \mathrm{~min}$. The RIE process conditions were: $\mathrm{SF}_{6}(50 \mathrm{sccm})$, chamber pressure $1.33 \mathrm{~Pa}$, bias voltage $100 \mathrm{~V}$, and radio-frequency power $200 \mathrm{~W}$. The resulting nanoroughness on the top surface of the PDMS microposts was characterized by AFM to be about $53 \mathrm{~nm}$.

Surface Functionalization of PDMS Micropost Array: As described previously, ${ }^{[24,25]}$ we used microcontact printing to functionalize the PDMS microposts with ECM proteins to promote cell attachment. Briefly, a flat 1:30 PDMS stamp was prepared and inked with fibronectin (BD Biosciences, San Jose, CA) at a saturating concentration of $50 \mathrm{mg} \mathrm{mL}^{-1}$ in distilled water for $1 \mathrm{~h}$ at room temperature. The PDMS stamp was then thoroughly rinsed with distilled water and blown dry with nitrogen gas. In parallel, the PDMS micropost array was treated with ultraviolet (UV) ozone (UV-ozone cleaner; Jelight, Irvine, CA) for $7 \mathrm{~min}$ to ionize the PDMS surface and thus facilitate transfer of ECM molecules from the stamp to the PDMS micropost tops. The fibronectin-coated PDMS stamp was then gently placed in conformal contact with the PDMS micropost array for $30 \mathrm{~s}$ to complete the protein transfer process. To utilize the PDMS micropost array for live-cell traction force measurements, we stained the PDMS microposts with $1,1^{\prime}$-dioleyl-3,3,3',3'-tetramethylindocarbocyanine methanesulfonate ( $\Delta^{9}$-Dil; Invitrogen). Pluronics F127 NF dissolved in PBS $(0.2 \%, w / v$; BASF, Ludwigshafen, Germany) was then adsorbed onto the PDMS surface for $1 \mathrm{~h}$ at room temperature to prevent protein adsorption to nonfunctionalized portions of the PDMS micropost array.

Quantification of Cellular Traction Force: Cell traction forces were quantified as previously described. ${ }^{[24,25]}$ In brief, phasecontrast images of live cells and fluorescence images of $\Delta^{9}$-Dilstained PDMS microposts underlying the cells were taken at the focal plane passing through the top surface of the posts with a $40 \times$ objective on the Zeiss Observer Z1 microscope attached to the AxioCam camera. The microscope was enclosed in the Carl Zeiss $\mathrm{XL} \mathrm{S1} \mathrm{environmental} \mathrm{chamber} \mathrm{to} \mathrm{maintain} \mathrm{the} \mathrm{experimental} \mathrm{envi-}$ ronment at $37{ }^{\circ} \mathrm{C}$ and $5 \% \mathrm{CO}_{2}$. Images were then analyzed with a custom-developed MATLAB program to calculate the deflection $\delta$ of the post centroid from its ideal position determined by the free and undeflected posts, which was then converted to the horizontal traction force $f$ using the expression $f=K \delta$, where $K$ is the nominal spring constant of the PDMS micropost calculated from the EulerBernoulli beam theory.

Statistics: The $p$-value was calculated using the Student $t$-test function in Excel (Microsoft, Seattle, WA). 


\section{Supporting Information}

Supporting Information is available from the Wiley Online Library or from the author.

\section{Acknowledgements}

We acknowledge financial support from the National Science Foundation (CMMI 1129611) and the Department of Mechanical Engineering at the University of Michigan, Ann Arbor. We thank M. Yang and C. S. Chen for sharing with us their MATLAB program to quantify cellular traction forces. The Lurie Nanofabrication Facility at the University of Michigan, a member of the National Nanotechnology Infrastructure Network (NNIN) funded by the National Science Foundation, is acknowledged for support in microfabrication.

[1] B. Geiger, J. P. Spatz, A. D. Bershadsky, Nat. Rev. Mol. Cell Biol. 2009, 10, 21.

[2] M. M. Stevens, J. H. George, Science 2005, 310, 1135.

[3] V. Vogel, M. Sheetz, Nat. Rev. Mol. Cell Biol. 2006, 7, 265.

[4] N. Sniadecki, R. A. Desai, S. A. Ruiz, C. S. Chen, Ann. Biomed. Eng. 2006, 34, 59.

[5] A. J. Engler, S. Sen, H. L. Sweeney, D. E. Discher, Cell 2006, 126, 677.

[6] D. E. Discher, P. Janmey, Y. L. Wang, Science 2005, 310, 1139.

[7] A. Curtis, C. Wilkinson, Biomaterials 1997, 18, 1573.

[8] V. Brunetti, G. Maiorano, L. Rizzello, B. Sorce, S. Sabella, R. Cingolani, P. P. Pompa, Proc. Natl. Acad. Sci. USA 2010, 107, 6264.
[9] A. Dolatshahi-Pirouz, T. Jensen, D. C. Kraft, M. Foss, P. Kingshott, J. L. Hansen, A. N. Larsen, J. Chevallier, F. Besenbacher, ACS Nano 2010, 4, 2874.

[10] R. J. Mannix, S. Kumar, F. Cassiola, M. Montoya-Zavala, E. Feinstein, M. Prentiss, D. E. Ingber, Nat. Nanotechnol. 2008, 3, 36.

[11] M. J. Dalby, N. Gadegaard, R. Tare, A. Andar, M. O. Riehle, P. Herzyk, C. D. W. Wilkinson, R. O. C. Oreffo, Nat. Mater. 2007, 6, 997.

[12] D. H. Kim, C. H. Seo, K. Han, K. W. Kwon, A. Levchenko, K. Y. Suh, Adv. Funct. Mater. 2009, 19, 1579.

[13] D. H. Kim, K. Han, K. Gupta, K. W. Kwon, K. Y. Suh, A. Levchenko, Biomaterials 2009, 30, 5433.

[14] T. P. Kunzler, C. Huwile, T. Drobek, J. Vörös, N. D. Spencer, Biomaterials 2007, 28, 5000.

[15] A. I. Teixeira, G. A. McKie, J. D. Foley, P. J. Berticsc, P. F. Nealey, C. J. Murphy, Biomaterials 2006, 27, 3945.

[16] C. Gonzalez-Garcia, S. R. Sousa, D. Moratal, P. Rico, M. Salmeron-Sanchez, Colloids Surf. B 2010, 77, 181.

[17] M. J. Dalby, S. Childs, M. O. Riehle, H. J. H. Johnstone, S. Affrossman, A. S. G. Curtis, Biomaterials 2003, $24,927$.

[18] K. L. Elias, R. L. Price, T. J. Webster, Biomaterials 2002, 23, 3279.

[19] M. Schindler, I. Ahmed, J. Kamal, A. Nur-E-Kamal, T. H. Grafe, H. Y. Chung, S. Meiners, Biomaterials 2005, 26, 5624.

[20] E. Metwalli, C. G. Pantano, Nucl. Instrum. Methods B 2003, 207, 21.

[21] P. W. Leech, Vacuum 1999, 55, 191.

[22] D. Y. Choi, J. H. Lee, D. S. Kim, S. T. Jung, J. Appl. Phys. 2004, 95, 8400.

[23] C. Hahn, M. A. Schwartz, Nat. Rev. Mol. Cell Biol. 2009, 10, 53.

[24] J. Fu, Y. K. Wang, M. T. Yang, R. A. Desai, X. Yu, Z. Liu, C. S. Chen, Nat. Methods 2010, 7, 733.

[25] M. T. Yang, J. P. Fu, Y. K. Wang, R. A. Desai, C. S. Chen, Nat. Protoc. 2011, 6, 187.

[26] S. N. Weng, J. P. Fu, Biomaterials 2011, 32, 9584.

[27] J. Garra, T. Long, J. Currie, T. Schneider, R. White, M. Paranjape, J. Vac. Sci. Technol. A 2002, 20, 975.

[28] M. T. Yang, N. J. Sniadecki, C. S. Chen, Adv. Mater. 2007, 19, 3119.

Received: May 20, 2012

Published online: August 7, 2012 